\begin{tabular}{l|l|l} 
Journal of the Egyptian Mathematical Society & ISSN: 1110-256x \\
Volume (26) - Issue (1) - 2018 & DOI: 10.21608/JOEMS.2018.9458
\end{tabular}

\title{
A SCALABLE PARALLEL ALGORITHM FOR TURNPIKE PROBLEM
}

\author{
Hazem M. Bahig ${ }^{1,2}$ and Mostafa M. Abbas ${ }^{3}$
}

\author{
${ }^{1}$ Computer Science Division, Department of Mathematics, Faculty of Science, Ain Shams \\ University, Cairo 11566, Egypt. hbahig@sci.asu.edu.eg, hazem.m.bahig@ gmail.com \\ ${ }^{2}$ Computer Science and Software Engineering Department, College of Computer Science and \\ Engineering, Hail University, Hail, KSA. \\ ${ }^{3}$ Qatar Computing Research Institute, Hamad Bin Khalifa University, Doha, Qatar. \\ mohamza@hbku.edu.qa
}

Received 8/1/2017 Revised 30/4/2017 Accepted 5/11/2017

\begin{abstract}
Given a set of all pairwise distances between points on a line. The Turnpike problem is to reconstruct the positions of those points. We reduce the running time to generate a set of solution for the turnpike problem by designing a parallel algorithm on a multicore system in case of the worst case. The algorithm is based on dividing the original problem into many independent subproblems of balanced size. The experimental study shows that our parallel algorithm significantly reduces the running time of the best practical sequential algorithm. Also, the scalability of the proposed algorithm is linear. Finally, we apply the proposed algorithm on restriction site mapping of DNA.
\end{abstract}

Keywords: turnpike, exact algorithm, parallel algorithm, multicore.

MSC: 65T05, 68W10, 68Q25

\section{Introduction}

Given a set of $n$ points, $A=\left(0=a_{1}, a_{2}, \ldots, a_{n}\right)$, on a line in increasing order, $\left(a_{1}<a_{2}<\ldots<a_{n}\right)$. The set of all pairwise differences between points in $A$ is denoted by $\Delta A=\left\{\left|a_{i}-a_{j}\right|: a_{i}, a_{j} \in A\right\}$. The size of the set of all pairwise differences between points is equal to $n(n-1) / 2$. The Turnpike reconstruction problem is to reconstruct a point set from the distances. Formally, we define the turnpike problem as follows [1].

Given a multiset, $\Delta A$, of $n(n-1) / 2$ positive numbers. Find a set $A$, if exist, such that $\Delta A$ is exactly the multiset of all positive pairwise differences of the elements of $A$.

The problem has many applications in different domains such as computational geometry [2], physics, especially in X-ray crystallography [3-4], and molecular biology, especially in restriction site mapping of DNA [5-7].

Finding an exact solution for turnpike problem is challenging due to the exponential time in the worst case. The exact algorithms for turnpike problem can be divided into impractical and practical algorithms. There are two impractical brute force algorithms and depend on two steps. The first step is to construct all sets of $n$-2 different integers from the set $Z$. The second step is to add the elements 0 and $M$ (Maximum element in $D$ ) for each set constructed in the Step 1 and check if this set represents a solution for turnpike problem or not. The difference between the two brute force algorithms is the elements of the set $Z$. One of the brute force algorithms takes $Z=\{x \mid 0<x<M\}$ and requires $O\left(M^{n-2}\right)$ time complexity. The other brute force algorithm takes $Z=\{x \mid 0<x<M$ and $x \in D\}$ and requires $O\left(n^{2 n-4}\right)$ time complexity. Lemek and Wermano proposed an impractical algorithm depends on polynomial factorization and requires $O\left(n^{M}\right)$ time complexity [8,9]. On the other hand, in [10], Skiena et al. presents a practical exact algorithm that depends on a branch and bound strategy. They proposed two bounding conditions to cut the search space. In the average case, the running time of the proposed algorithm is $\mathrm{O}\left(n^{2} \log n\right)$. In [11], the author proved that the algorithm takes non-polynomial time in the worst case for certain types of instances. In 2016, Fomin [12] introduced an exact solution which is faster than Skiena, Smith, and Lemke algorithm, SSL, [10] for some instances, while SSL algorithm is better than Formin's algorithm in the class of worst case.

The approach in this research paper is to design a parallel algorithm based on SSL algorithm under a multi-core architecture. The proposed algorithm is based on dividing the original problem into many independent subproblems of equal size. Subsequently, we can solve each subproblem using SSL algorithm. Another subgoal is the sequential part of the proposed algorithm required very short time. We apply our method to a class of instances that meet the worst case and prove that the 
running time of the proposed parallel algorithm decreases with an increase in the number of processors. We also show that the scalability of the parallel algorithm is linear.

The remainder of this paper is as follows. In Section 2, we describe SSL algorithm as a recursive algorithm and then convert it into an iterative algorithm. In Section 3, we propose an efficient parallel algorithm on a multicore system. In Section 4, we present the experimental results on a class of instances that meet the worst case. We also measure the running time, speedup, scalability and storage of our method. In Section 5, we apply the proposed algorithm on molecular biology filed, especially on restriction site mapping of DNA. We conclude in Section 6.

\section{SSL Algorithm}

In this section, we give an overview of SSL algorithm [10], which is used to find the solution of turnpike problem. The main idea behind SSL algorithm is to construct a set $X$ as follows:

- $\quad$ Find the largest element in the set $D$, say $y$, using the procedure Find_Max.

- For each value of $\delta$, test if the value of $\delta$ can be a part of the solution $X$. If the condition is true then add the value of $\delta$ to $X$ and delete all values of $\Delta(\delta, X)$ from $D$, where:

$\circ \delta=y$ and $\delta=$ width $-y$.

○ width is the maximum value of the initial value of the set $D$.

$\circ \quad$ the value of $\delta$ can be a part of the solution $X$ if the multiset, $\Delta(\delta, X)$, of distances between $\delta$ and all values in the set $X$ is a subset of $D$.

$\circ \Delta(\delta, X)=\{|\delta-y|: \forall y$ in $\mathrm{X}\}$.

- $\quad$ Repeat the previous steps until the set $D$ is empty for each value of $\delta$.

These steps can be represented as procedure Place. The inputs of the procedure are two sets, $D$ and $X$. The goal of this procedure is to construct the set of solution $S$ that contains all values of $X$ such that $\Delta X=D$. The pseudocode for the algorithm is as follows [10].

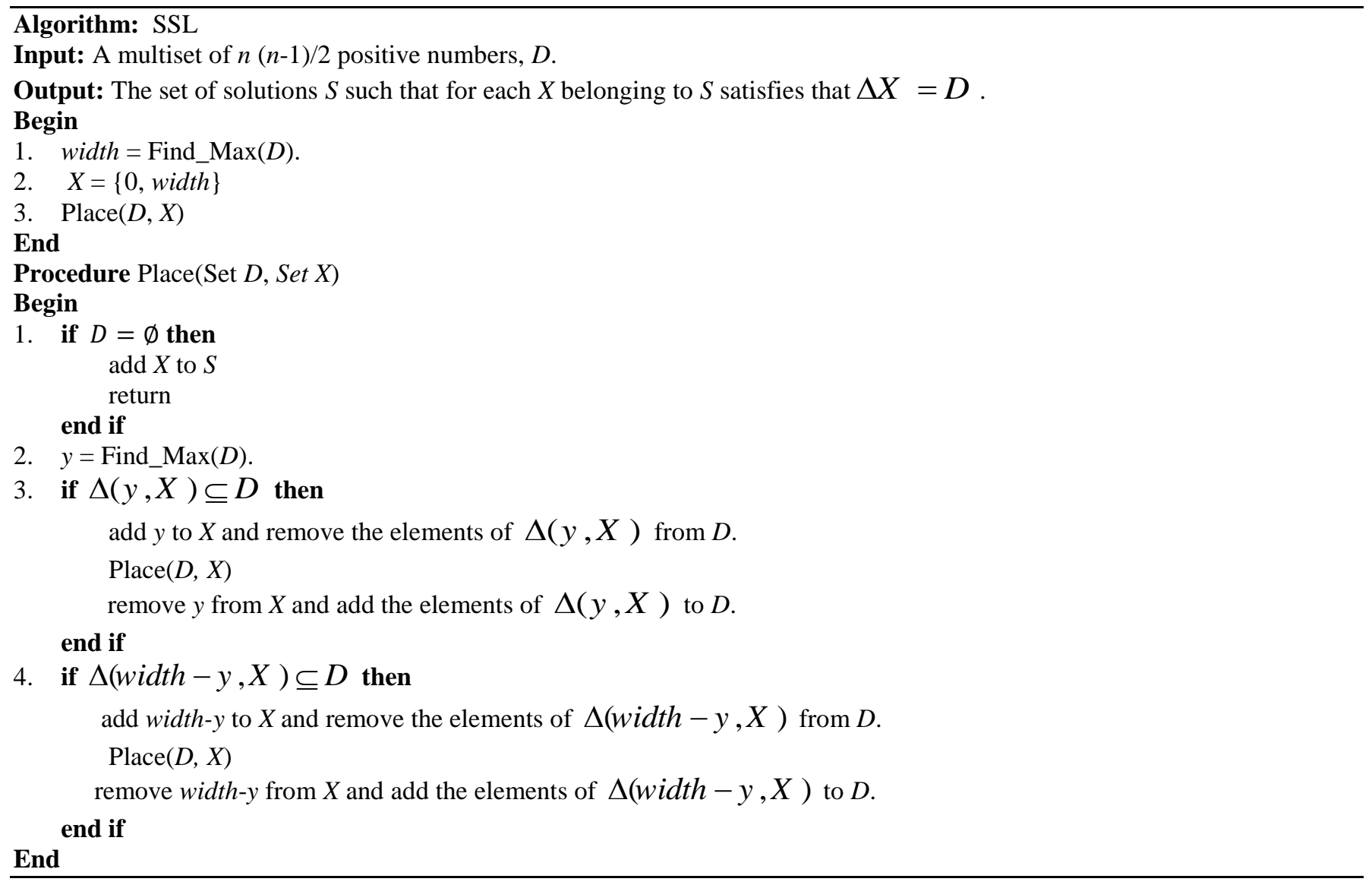

SSL algorithm works with polynomial running time $\mathrm{O}\left(n^{2} \log n\right)$ if there is no backtracking. The backtrack came from the last statement in Step 3 and 4 in the procedure Place $(D, X)[10]$. The recursion in the procedure Place $(D, X)$ can be represented as a tree with depth $<n$ and degree $\leq 2$. In the worst case, the depth and degree of the tree are $n-1$ and 2 respectively. This implies that the number of recursive calls for the procedure Place $(D, X)$ is $O\left(2^{n-1}\right)$. In [11], the author defines the class of instances that satisfy the worst case by using the proposition in [11].

Before parallelizing SSL algorithm, we will first rewrite the algorithm as an iterative algorithm by using stack. Each element of the stack contains a pair of sets $D$ and $X$. Initially, we push the two input sets $D$ and $X$ to the stack $T$. In each iteration we pop an element $e$ from the stack and do the same steps of procedure Place $(D, X)$, except the recursive call step that is replaced by 
pushing the element e to the stack. The algorithm will repeat the same steps on each popped element $e$ until the stack is empty. The pseudo-code of the iterative version for SSL algorithm is as follows.

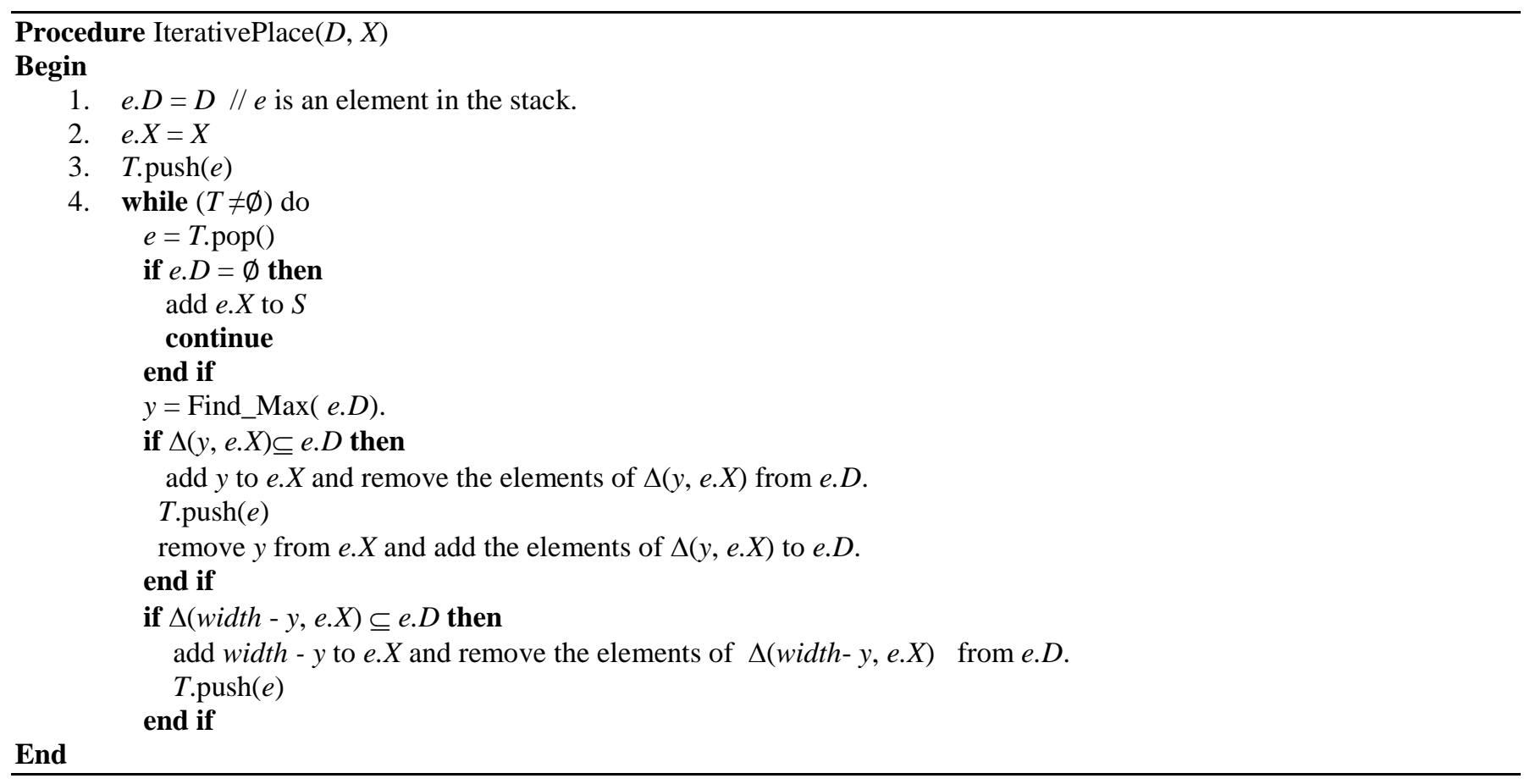

\section{Method of Parallelization}

In this section, we parallelize the iterative algorithm for turnpike problem. To make the parallelization efficient we must verify the following characteristics from the proposed algorithm.(1) The subproblems generated by dividing the original problem are independent; (2) The subproblems are of equal size (according to the set $X$ ) ; and (3) The time required for the sequential part is minimal.

The main idea of parallelizing is based on procedure Divide. The procedure was designed to verify the two main objectives (1 and 2) stated previously. The input of the Divide procedure is a stack $T$. Each element of the stack consists of two sets, $D$ and $X$. The output of the procedure is the same stack with different elements. Each element of the output stack consists of two sets, $D$ and $X$, such that the size of the set $D$ in the output stack is less than the size of $D$ in the input stack. The goal of the Divide procedure is to generate at most two new elements from each element in $T$. In more details, if we have the element $e=(D, X)$ $\in T$ at the top of the stack, then the Divide procedure will pop the element $e$ from $T$ and push at most two elements onto the output stack according to one of the following cases:

Case 1: Only one new element will be pushed to the stack from either of the following: (i) $e^{\prime}=(D \backslash(y, X), X \mathrm{U}\{y\})$ if $\Delta(y, X) \subseteq$ $D$; or (ii) $\mathrm{e} "=(D \backslash($ width $-y, X), X \mathrm{U}\{$ width- $y\})$ if $\Delta($ width $-y, X) \subseteq D$.

Case 2: The two new elements will be pushed to the stack, $e^{\prime}$ and $e^{\prime \prime}$.

Case 3: No new element will be pushed to the stack.

In general, if we have $n_{1}$ elements in $T$ then the procedure terminates after it has popped $n_{1}$ elements from the stack $T$. The pseudo-code for the Divide procedure is as follows.

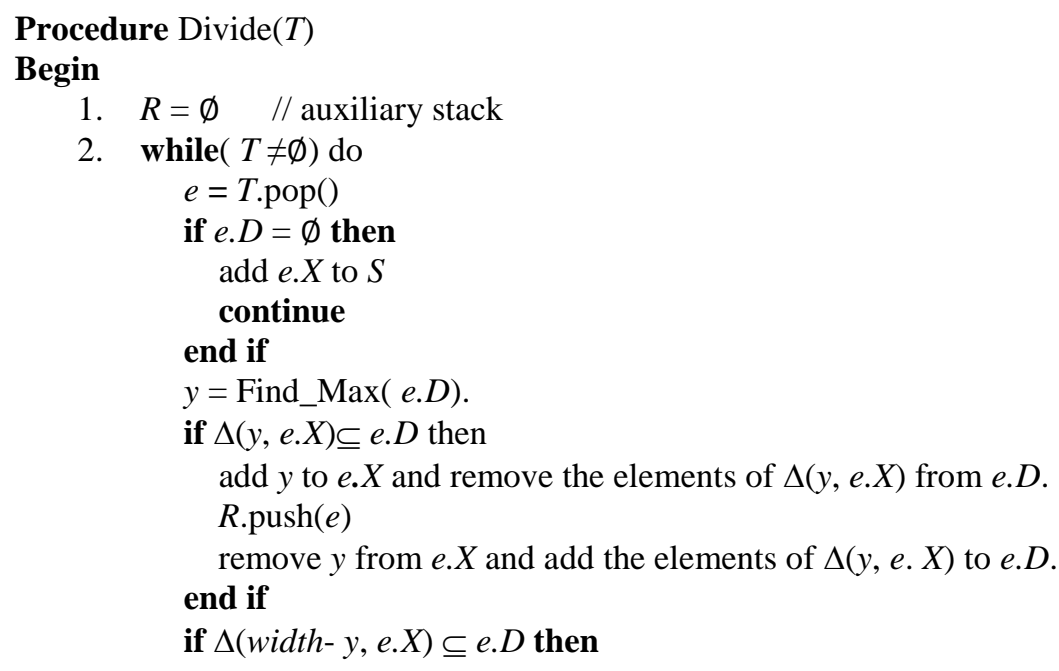


add width-y to $e . X$ and remove the elements of $\Delta($ width $-y, e . X)$ from e.D.

$$
R \cdot \operatorname{push}(e)
$$

3. $T=R$

end if

4. $R=\varnothing$

The two procedures, IterativePlace and Divide, are similar in their structure but differ in their behavior. The main differences between these two procedures are:

(1) In procedure IterativePlace, we do the process on the top of the stack and the results are pushed on the same stack, while in procedure Divide, we do the same process on the top of the stack and the results are pushed to the auxiliary stack $R$. After we have popped all elements from the stack $T$, the stack $R$ is assigned to $T$.

(2) If the size of stack $T$ is $n$ then the while-loop in procedure Divide will be finished after only $n$ iterations, whereas the whileloop in procedure IterativePlace requires more than $n$ iterations in general.

(3) The size of set $X$ for each element in stack $T$ after calling procedure Divide is equal, while the size of the set $X$ for each element in the stack $T$ after each iteration of the while-loop in procedure IterativePlace is different in the general case.

Figures 1 and 2 show the value of the stack $T$ on $D=\{1,3,4,6,7,7,10,13,13,17,20,21,22,24,25,28,29,34,35,41,42\}$ when using procedure IterativePlace and Divide respectively. Figure 1(a) represents the execution of the first three steps of procedure IterativePlace. Figures 1(b-d) represent the execution of the first, second, and third iterations of the while-loop for procedure IterativePlace respectively. Figure 2(a) represents the value of the stack $T$ before executing procedure Divide. Figures $2(b-d)$ represent the value of the stack T after the first, second, and third call of procedure Divide respectively.

Now, we return to the main objective of this section, which is parallelizing SSL algorithm. We can summarize the main steps of parallelization as follows:

1. Initially, we create stack $T$ containing one element, $(D, X)$, where $D$ is the input of the problem and $X=\{0$, width $=$ $\operatorname{Maximum}(D)\}$. The set of solutions, S, is empty. Also, for each processor, $p_{i}$, assign an empty set to the subsolution set, $S_{i}$, $\forall 0 \leq i<P$. This step represents the lines 1-9 in P-SSL algorithm.

2. If the number of elements in the stack is greater than or equal to $P$ then we do the following (see the lines 11-15 in P-SSL algorithm):

a. Pop the first $P$ elements from stack $T$ and assign each one to a processor.

b. Each processor, $p_{i}$, works on one of the popped elements using the sequential procedure IterativePlace to find the solution, if it exists, and then adds this to $S_{i}$.

3. Otherwise (if the number of elements in the stack is less than $P$ ), we apply the Divide procedure, see the lines $16-18$ in $\mathrm{P}$ SSL algorithm.

4. Repeat the Steps 2-3 if the stack is not empty, see the line 10 in P-SSL algorithm.

5. Combine the set of solutions generated from the $P$ processors to the set $S$, see the line 20 in P-SSL algorithm.

6. Remove the duplicates from the set $S$, see the line 21 in P-SSL algorithm.

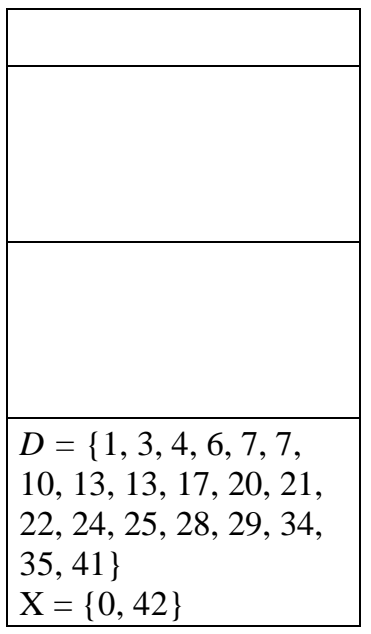

(a)

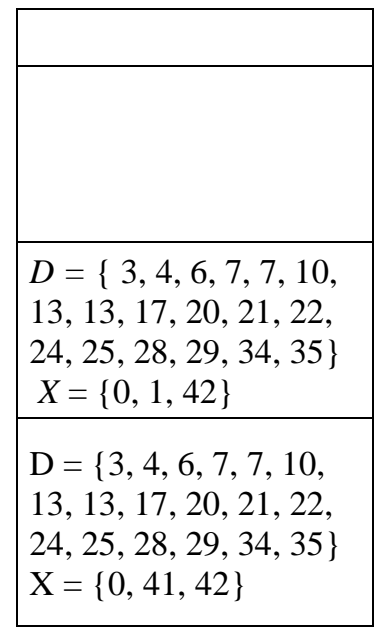

(b)

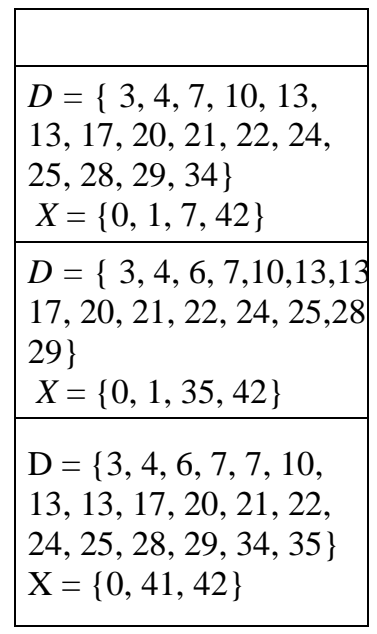

(c)

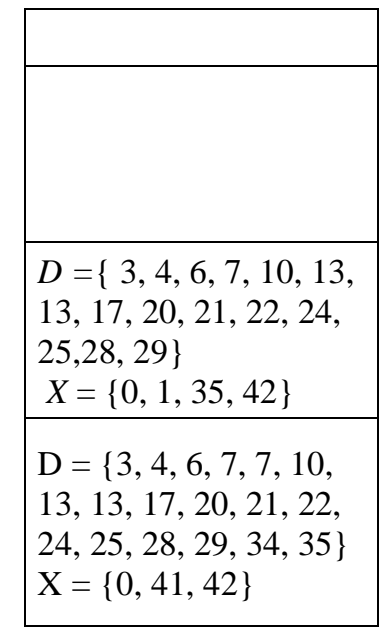

(d)

Figure 1: Value of stack $T$ after executing 3 iterations in IterativePlace procedure 


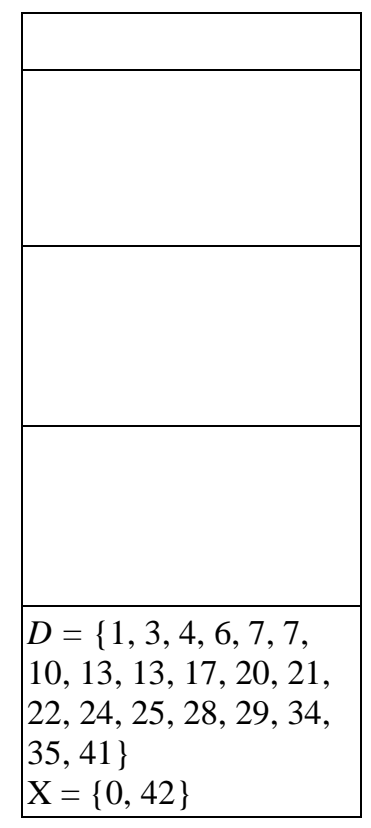

(a)

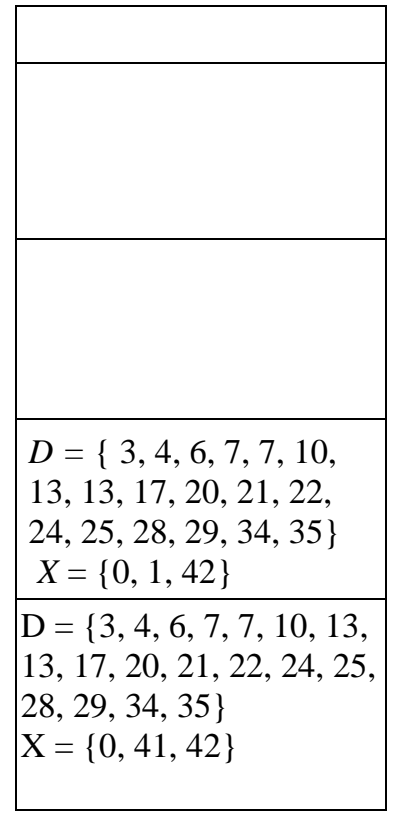

(b)

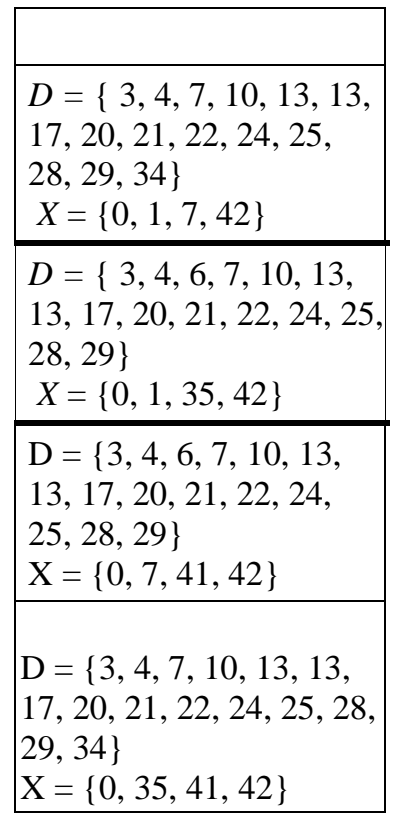

(c)

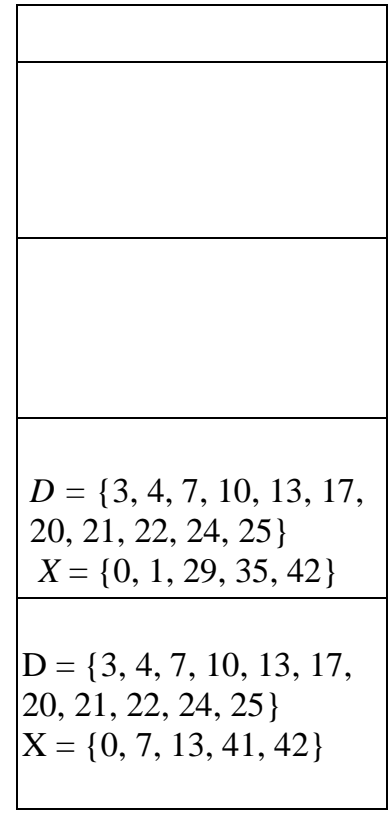

(d)

Figure 2: Value of stack $T$ after calling Divide procedure 3 times.

The pseudo-code for parallelizing SSL algorithm, P- SSL, is as follows.

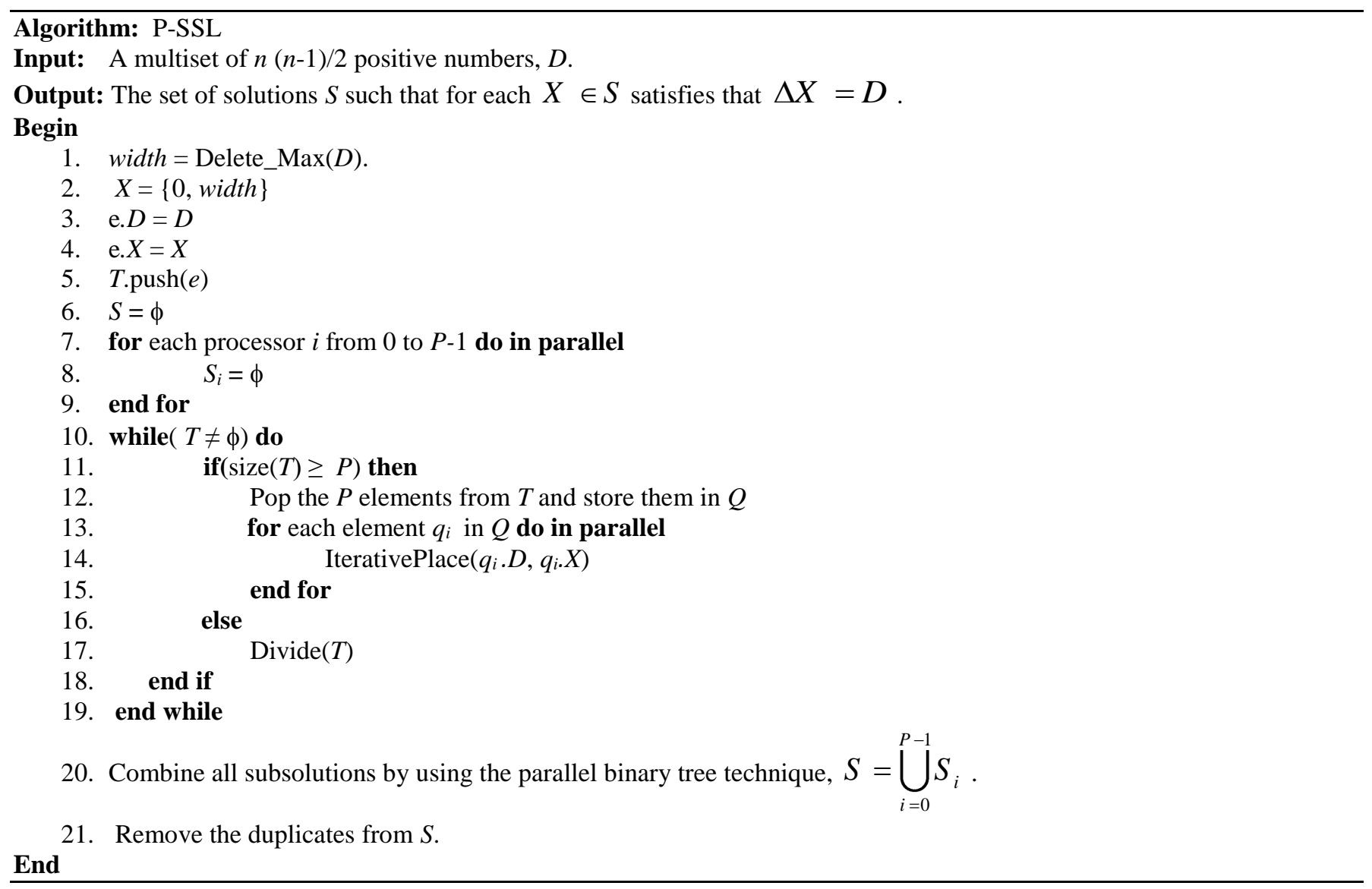

In order to compute the complexity analysis of the proposed algorithm, we assume that $P=2^{\alpha}$ processors. The worst case happens when each node (problem) in the solution tree produces two nodes (subproblems). Therefore, in the worst case we will have $P=2^{\alpha}$ nodes at the level $\alpha$ of the solution tree. The time complexity of the P-SSL to produce the $2^{\alpha}$ nodes at the level $\alpha$ is $O\left(2^{\alpha} n \log \alpha\right)=O(n P \log P)$; while the memory complexity is $O\left(2^{\alpha} n^{2}\right)=\mathrm{O}\left(n^{2} P\right)$. After obtaining the $P=2^{\alpha}$ nodes on the solution tree, the P-SSL will assign one node to a processor. The time complexity of any processor to solve its task is $O\left(2^{n-\alpha} n \log (n-\right.$ $\alpha))=O\left(\left(n 2^{n} \log (n-\log P)\right) / P\right)$, while the memory complexity is $O\left((n-\alpha) n^{2}\right)=O\left((n-\log P) n^{2}\right)$. The time complexity of the P-SSL 
is $O\left(n 2^{\alpha}\left(\log \alpha+2^{n-2 \alpha} \log (n-\alpha)\right)\right)=\mathrm{O}\left(n P\left(\log \log P+2^{n-2 \log P} \log (n-\log P)\right)\right)$; while the memory complexity is $O\left(n^{2}\left(2^{\alpha}+(n-\alpha)\right)\right)=$ $O\left(n^{2}(P+(n-\log P))\right)$. The cost of the P-SSL is $O\left(n P^{2}\left(\log \log P+2^{n-2 \log P} \log (n-\log P)\right)\right)$.

\section{Results and Discussion}

In this section, we present our experiments for SSL algorithm and its parallelization, P-SSL. We will evaluate the performance of our proposed parallel algorithm by comparing it with the sequential algorithm on simulated data in terms of the running time and storage. We also measure the scalability of the proposed parallel algorithm.

To verify these goals, we implemented the algorithms on a Dual Octa-core processors machine (Intel Xeon E5-2690) with 128 GB RAM. Each processor has a speed of $2.9 \mathrm{GHz}$ with a $20 \mathrm{MB}$ Cache. The algorithms codes are compiled using the -O3 flag in g++ under 64-bit enterprise Linux Red Hat 4.4 and implemented by C++ language and openMP directives.

In our experimental study we use Zhang instances [11]. The reason for selecting this kind of data is that SSL algorithm runs in exponential time on this data. The instances are generated by creating a set $A$ according to Zhang instances, and then we calculate the set $D=\Delta A$.

The first criteria used to measure the performance of the proposed algorithm is the running time. In our experimental study we use a different number of cores $P=2,4,6, \ldots$, and 16 . For each instance of size $n$, the running time of the proposed parallel algorithm is equal to the average time for $t$ different cases, where $t$ is given in Table 1 . We use $s, m$ and $h$ to represent the time in seconds, minutes and hours, respectively.

\begin{tabular}{|c|c|c|c|}
\hline$n$ & $n<40$ & $40 \leq n<60$ & $n=60$ \\
\hline$t$ & 20 & 10 & 5 \\
\hline
\end{tabular}

Table 1: number of instances for different values of $n$.

Table 2 shows the running time of SSL and P-SSL algorithms. From the tables, we observe that the running time of SSL algorithm increases with an increase in the value of $n$. The running time of SSL algorithm for $n=55$ required approximately 1 day; while the algorithm required more than 4 days in case of $n=60$. Therefore, SSL algorithm is not practical for large value of $n$ in the worst case.

\begin{tabular}{|c|c|c|c|c|c|c|c|c|c|}
\hline$P$ & $n=20$ & $n=25$ & $n=30$ & $n=35$ & $n=40$ & $n=45$ & $n=50$ & $n=55$ & $n=60$ \\
\hline 1 & 0.141 & 1.255 & 8.521 & 64.888 & 391.8 & 48.355 & 241.08 & 1327.62 & 6318.48 \\
\hline 2 & 0.088 & 0.827 & 4.829 & 33.507 & 196.98 & 24.671 & 128.58 & 692.34 & 3251.22 \\
\hline 4 & 0.044 & 0.317 & 2.435 & 17.598 & 95.28 & 12.684 & 62.7 & 349.44 & 1627.32 \\
\hline 6 & 0.029 & 0.225 & 1.641 & 11.729 & 68.88 & 8.828 & 43.02 & 233.64 & 1088.64 \\
\hline 8 & 0.021 & 0.167 & 1.208 & 8.69 & 52.56 & 6.277 & 31.44 & 178.8 & 832.14 \\
\hline 10 & 0.018 & 0.141 & 1.019 & 7.229 & 42.24 & 5.226 & 26.7 & 142.56 & 666.6 \\
\hline 12 & 0.016 & 0.119 & 0.854 & 6.063 & 35.64 & 4.383 & 21.9 & 120 & 588.66 \\
\hline 14 & 0.014 & 0.102 & 0.719 & 5.187 & 30.72 & 3.743 & 18.36 & 101.22 & 484.74 \\
\hline 16 & 0.029 & 0.107 & 0.642 & 4.525 & 26.82 & 3.245 & 16.56 & 86.34 & 416.04 \\
\hline
\end{tabular}

Table 2: Running time of SSL and P-SSL algorithms for instances $20 \leq n \leq 60$.

Also the results show that the running time for the P-SSL algorithm decreases with increase in the number of cores, as shown in Figures 3 and 4. In these two figures we use the log scale to represent the running time of P-SSL. We have two exception cases that occur when $n$ is small and $P$ is large. In case of $n=20$ and $\mathrm{P}=16$, the running time of the P-SSL algorithm is greater than the running time of the P-SSL algorithm when $P=14,12,10$, and 8. Also, in case of $n=25$ and $P=16$ the running time of P-SSL algorithm is greater than the running time of P-SSL algorithm when $P=14$. These exceptional cases can be accounted for by the fact that the running time for SSL algorithm is very small. So, the parallelization is not efficient with a large number of cores. Also, in some cases, especially when $n$ is small, we cannot divide the original problem into $P=16$ subproblems.

Additionally, the percentage of improvement in the running time for the P-SSL algorithm increases with an increase in the number of cores. For example, the percentages of improvement when $n=60$ are $48.5 \%, 74,2 \%, 82.8 \%, 86.8 \%, 89.4 \%, 90.7 \%$, $92.3 \%$, and $93.4 \%$ in case of $P=2,4,6,8,10,12,14$, and 16 respectively. Also, we show that for a fixed value of $n$, the running time for the P-SSL algorithm is faster than for SSL algorithm.

The second criteria used to measure the performance of the proposed algorithm is the scalability of the algorithm. Figures 5 and 6 represent the scalability of the parallel algorithm as a function of the number of cores, $P$, and problem size $n$. The speedup for each instance $n$ is equal to the average of the speedup of the different cases of this $n$, where $\operatorname{speedup}=T_{1} / T_{p}$. Figure 5 and 6 prove that the speedup achieved scales 


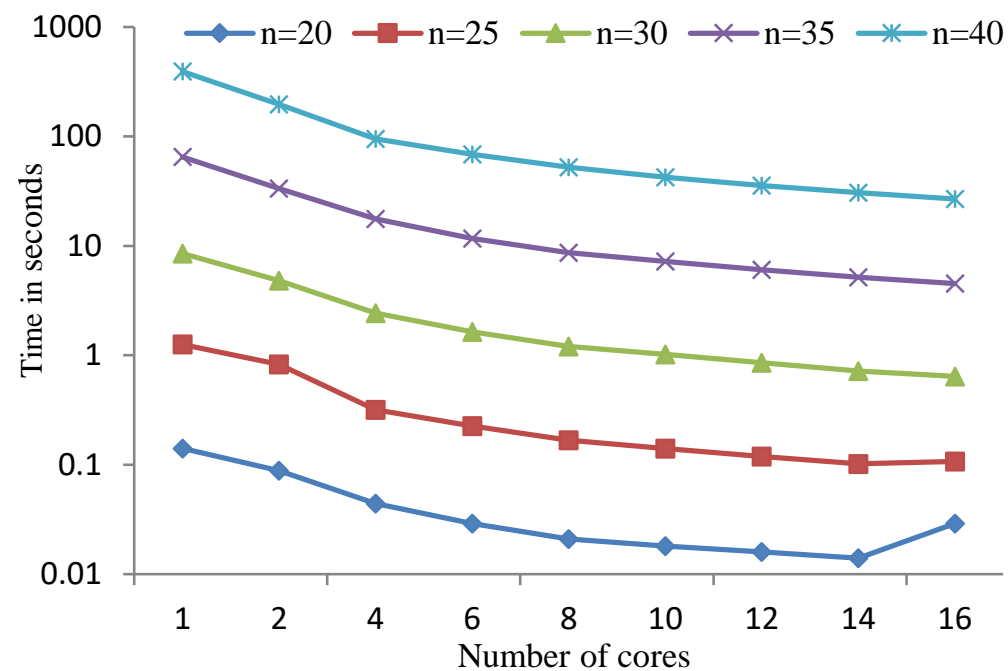

Figure 3: Running time behavior of P-SSL algorithm using different number of cores for instances $20 \leq n \leq 40$.

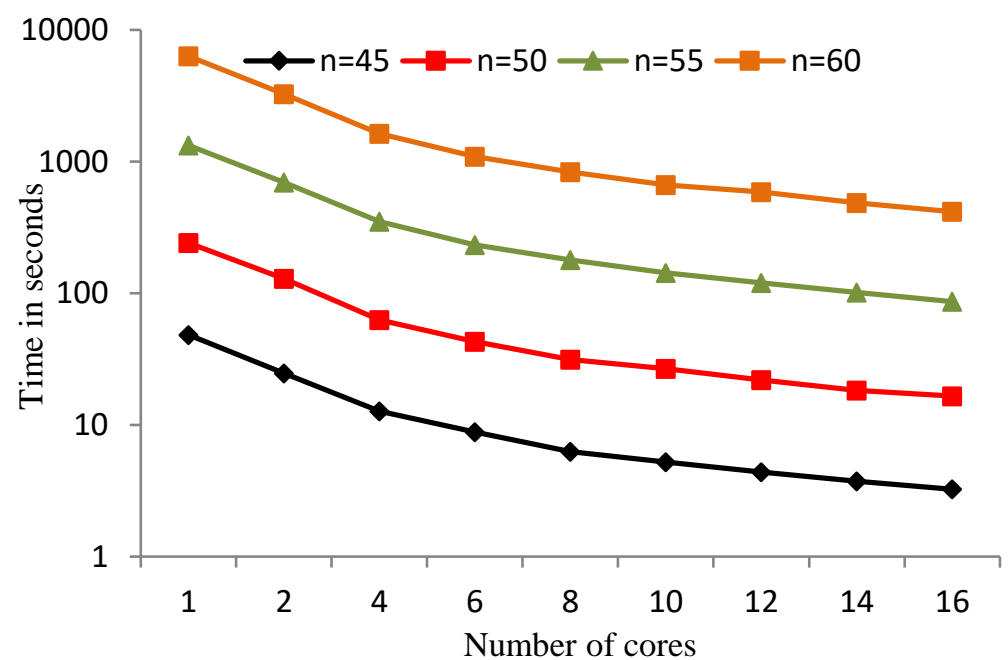

Figure 4: Running time behavior of P-SSL algorithm using different number of cores for instances $45 \leq n \leq 60$.

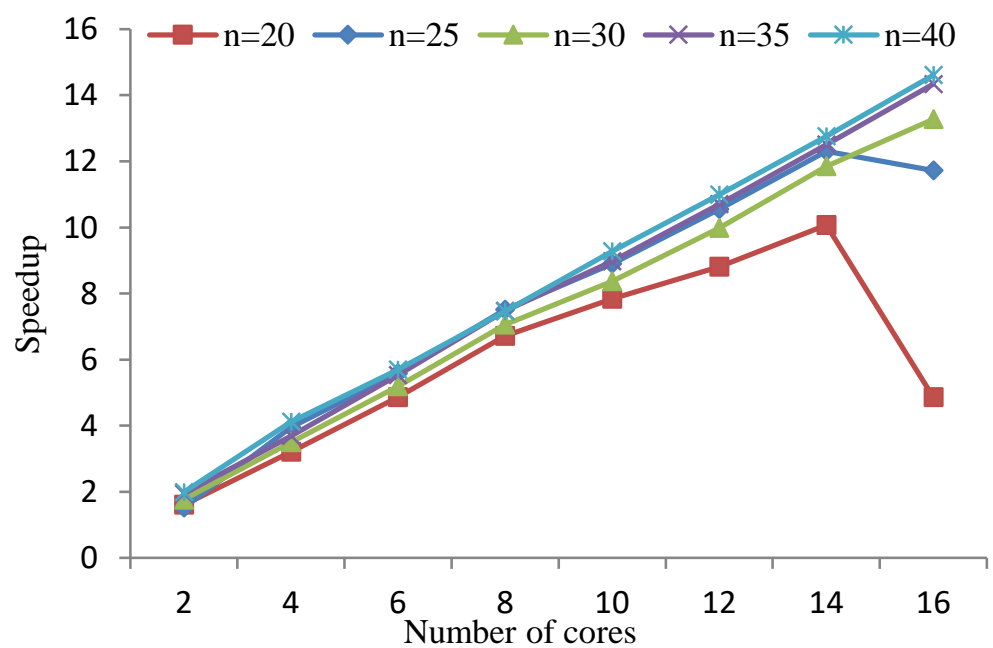

Figure 5: Scalability of P-SSL algorithm in case of $20 \leq n \leq 40$. 


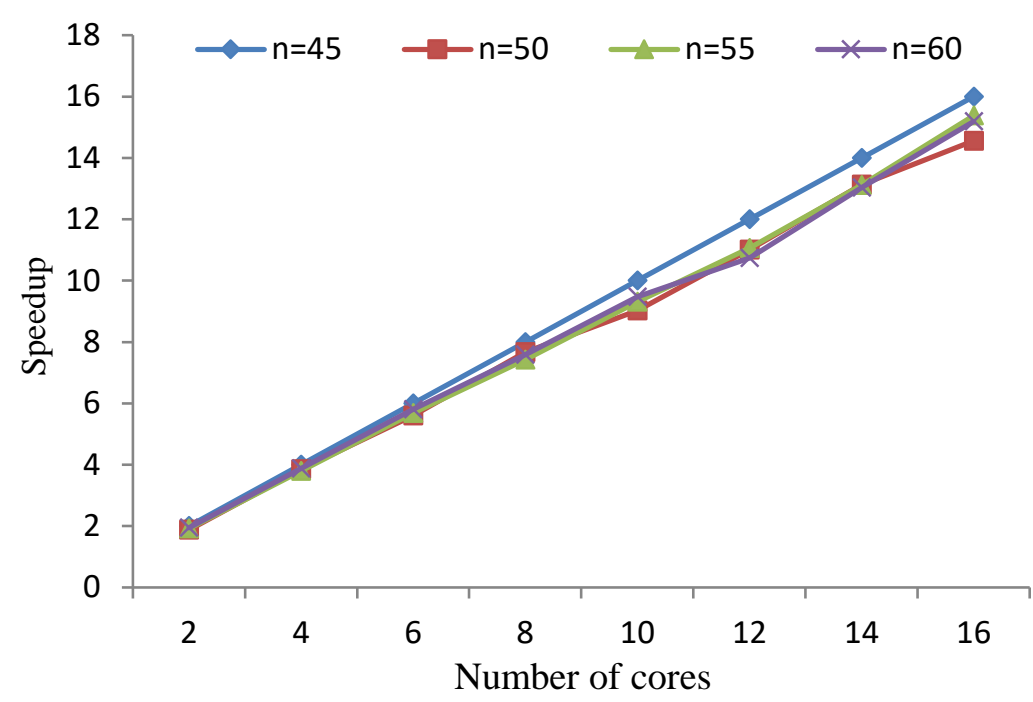

Figure 6: Scalability of P-SSL algorithm in case of $45 \leq n \leq 60$.

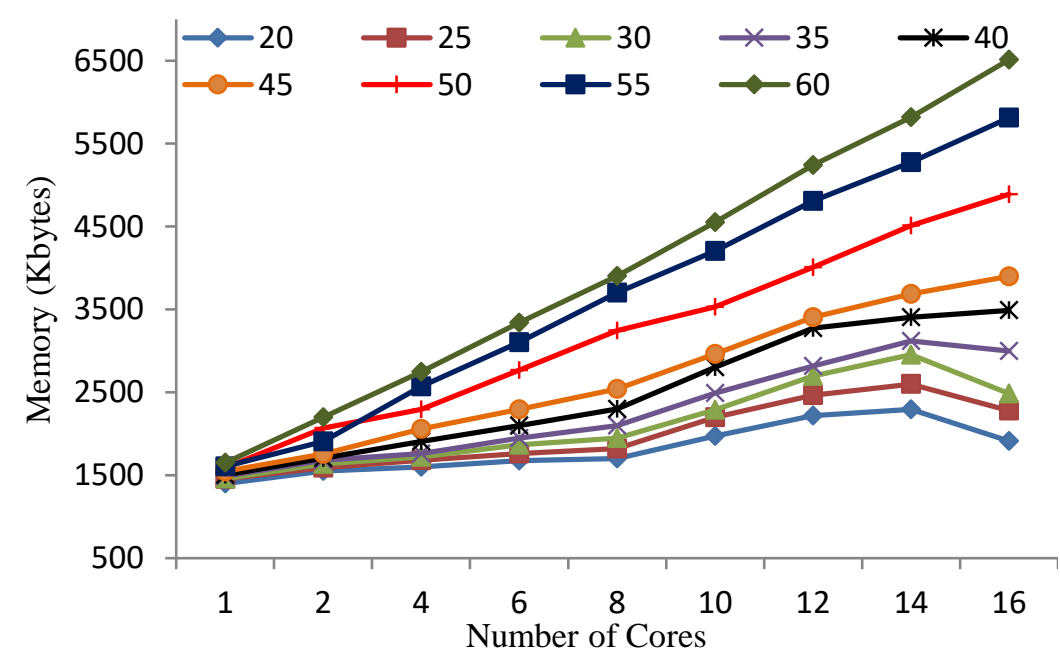

Figure 7: Storage of P-SSL for different values of $n$ and $P$.

linearly with an increase in the number of cores. In case of $n=20$, the speedup decreases with a large number of cores, because the running time at $P=16$ is not large (as discussed in the first criteria). So, the value of speedup is smaller than $P \leq 14$.

The third criteria used to measure the performance of the proposed algorithm is the storage of the algorithm, which is total memory required by the algorithm. Figure 7 shows the storage comparison between SSL algorithm and the P-SSL algorithm.

It is clear that for fixed value of $n$, the storage of the P-SSL algorithm increases with an increase in the number of cores. The reason for increasing the storage of the P-SSL algorithm is that the $P$ cores work on $P$ different subproblems at the same time. Each core has auxiliary storage for each subproblem. So, the total storage of the P-SSL algorithm will be increased. In some cases, we cannot divide the problem into $P$ subproblems, especially when $n$ is small and $P$ is large. So, the number of active cores is less than $P$. Therefore, the storage of the P-SSL algorithm will be decreased. For example $n=20,25$, and 30 and $P=16$.

\section{Application to Molecular Biology}

In this section, we applied the proposed parallel algorithm, P-SSL, on the field of molecular biology, especially in restriction site mapping of DNA. In our experiment, we use three genomes and two restriction enzymes for each. The three genomes are Bacillus subtilis subsp. subtilis str. 168, Ecoli IAI39 and E. Coli K12 genomes [13,14], while the restriction enzymes are NotI, and XbaI [15]. The size of Bacillus subtilis subsp. subtilis str. 168, E. Coli IAI39 and E. Coli K12 genomes is $4.02 \mathrm{M} \mathrm{bp,} 4.89 \mathrm{M}$ bp, and $4 \mathrm{M}$ bp respectively.

We first apply the proposed algorithm, P-SSL, on $D$ to generate the set of the solutions, $S=\left\{S_{1}, S_{2}, \ldots, S_{k}\right\}$. After that, the set, $S_{i}=\left\{s_{i 1}, s_{i 2}, \ldots, s_{i n i}\right\} \in S$ is a correct solution if the pattern (covers restriction sites) appears at each position, $s_{i j} \in S_{i}$, in the genome is similar. Otherwise, we discard the set $S_{i}$. We can do this step by pattern matching algorithm.

Table 3 represents the application of our work on three genomes. The first column in the table is the restriction enzymes used in the restriction site mapping DNA. For each genome, we have two columns: the first column represents the input size of the PSSL algorithm, while the second column represents the output size of the P-SSL algorithm for each set in the set of the solutions. 
The last two columns in the table represent the name of the restriction site in the genome detected by the algorithm and published respectively. The output of the proposed parallel algorithm is similar to the restriction site that is published in GenBank [14] and $[15]$.

\begin{tabular}{|c|c|c|c|c|c|c|c|c|}
\hline \multirow{3}{*}{$\begin{array}{c}\text { Restriction } \\
\text { enzymes }\end{array}$} & \multicolumn{6}{|c|}{ Genome Type } & \multirow{3}{*}{$\begin{array}{c}\text { Detected } \\
\text { Restriction site }\end{array}$} & \multirow{3}{*}{$\begin{array}{c}\text { Published } \\
\text { Restriction site } \\
{[15]}\end{array}$} \\
\hline & \multicolumn{2}{|c|}{$\begin{array}{c}\text { Bacillus subtilis } \\
\text { subsp. subtilis str. } \\
168 \\
\end{array}$} & \multicolumn{2}{|c|}{ E. Coli IAI39 } & \multicolumn{2}{|c|}{ E. Coli K12 } & & \\
\hline & $|D|=N$ & $\left|S_{i}\right|=n$ & $|D|=N$ & $\left|S_{i}\right|=n$ & $|D|=N$ & $\left|S_{i}\right|=n$ & & \\
\hline NotI & 325 & 26 & 3486 & 84 & 780 & 40 & GCGGCCGC & GCGGCCGC \\
\hline XbaI & 528 & 33 & 39903 & 83 & 1891 & 62 & TCTAGA & TCTAGA \\
\hline
\end{tabular}

Table 3: Applying the proposed algorithm on Bacillus subtilis subsp. subtilis str. 168, Ecoli IAI39 and E. Coli K12.

\section{Conclusion}

Here we addressed one of the challenging problems which is the turnpike problem. The running time for the best known sequential algorithm, SSL, is exponential in the worst case. We proposed an efficient, scalable, parallel algorithm for turnpike problem. The algorithm is based on decomposing the original problem into many independent subproblems of equal size and then assigning each of these to a single processor. The experimental results on a multicore system show that the running time of the algorithm deceases with an increase in the number of cores. Additionally, the percentage of improvements in the running time increases with the number of cores. We also show that the parallel algorithm scales linearly. Finally, we applied the proposed algorithm on modular biology field.

\section{References}

1. T. Dakic. On the turnpike problem. PhD thesis, Simon Fraser University, 2000.

2. M. I. Shamos. Problems in computational geometry. Unpublished manuscript, 1975, Carnegie Mellon University, Pittsburgh, PA.

3. A. L Pattersan. A direct method for determination of the components of interatomic distances in crystals, Zeitschr. Krist. 90 (1935) 517-542.

4. A. L Pattersan. Ambiguities in the X-ray analysis of crystal structure, Php. Review. 65 (1944) 195-201.

5. J. Błażewicz, P. Formanowicz, M. Kasprzak, M. Jaroszewski, and W. T. Markiewicz. Construction of DNA restriction maps based on a simplified experiment. Bioinformatics. 17(5) (2001) 398-404.

6. L. Goldstein, and M. S. Waterman. Mapping DNA by stochastic relaxation. Advances in Applied Mathematics. 8(2) (1987) 194-207.

7. G. Pandurangan, and H. Ramesh. The restriction mapping problem revisited. Journal of Computer and System Sciences. 65(3) (2002) 526-544.

8. N. C. Jones, and P.A Pevzner. An introduction to bioinformatics algorithms. MIT Press, 2004.

9. P. Lemke, and M. Werman. On the complexity of inverting the autocorrelation function of a finite integer sequence, and the problem of locating $\mathrm{n}$ points on a line, given the $\left({ }^{\mathrm{n}} \mathrm{C}_{2}\right)$ unlabelled distances between them. Technical Report \# 453, IMA, Minneapolis 1988.

10. S. Skiena, W. D. Smith, and P. Lemke. Reconstructing sets from interpoint distances. In: Discrete and Computational Geometry. Edited by Aronov B, Basu S, Pach J, and Sharir M, Springer. 25 (1990) 597-631.

11. Z. Zhang. An exponential example for a partial digest mapping algorithm. Journal of Computational Biology. 1( 3) (1994) 235-239.

12. F. Eduard. A Simple approach to the reconstruction of a set of points from the multiset of $\mathrm{n} 2$ pairwise distances in $\mathrm{n} 2 \mathrm{steps}$ for the sequencing problem: II Algoirthm. Journal of Computational Biology. 23 (2016) 1-7.

13. DA. Benson, Dennis A., Mark Cavanaugh, Karen Clark, Ilene Karsch-Mizrachi, David J. Lipman, James Ostell, and Eric W. Sayers (2013). GenBank. Nucleic acids research 41, no. D1, D36-D42.

14. NCBI: https://www.ncbi.nlm.nih.gov/

15. https://en.wikipedia.org/wiki/Restriction_enzyme 